\title{
Mapping the intersection of language and reading: the neural bases of the primary systems hypothesis
}

\author{
Anna M. Woollams ${ }^{1}$ - Ajay Halai ${ }^{1}$ Matthew A. Lambon Ralph ${ }^{1}$
}

Received: 11 April 2017 / Accepted: 10 July 2018 / Published online: 2 August 2018

(c) The Author(s) 2018

\begin{abstract}
The primary systems framework has been used to relate behavioural performance across many different language activities to the status of core underpinning domain-general cognitive systems. This study provided the first quantitative investigation of this account at both behavioural and neural levels in a group of patients with chronic post-stroke aphasia. Principal components analysis was used to distil orthogonal measures of phonological and semantic processing, which were then related to reading performance and the underlying lesion distributions using voxel-based correlational methodology. Concrete word reading involved both a ventral semantic pathway, and inferior and anterior aspects of the dorsal phonological pathway. Abstract word reading overlapped with the ventral semantic pathway but also drew more extensively on the superior and posterior aspects of the dorsal phonological pathway. Nonword reading was related to phonological processing along the dorsal pathway and was also supported by a more superior set of regions previously associated with speech motor output. The use of continuous measures of behavioural performance and neural integrity allowed us to elucidate for the first time both the lesion and behavioural correlates for the semantic and phonological components of the primary systems hypothesis and to extend these by identifying the importance of an additional dorsal speech motor output system. These results provide a target for future neuroanatomically constrained computational models of reading.
\end{abstract}

Keywords Dyslexia $\cdot$ Aphasia $\cdot$ Phonology $\cdot$ Semantics $\cdot$ Speech

\section{Introduction}

Higher cognition is supported by a complex network of interacting brain regions. A great deal of neuropsychological and neuroimaging research has been devoted to isolation of individual task-specific aspects of this system. Yet, in an efficient system, much of the processing for any given task will be shared across more domain-general areas. To fully understand how the distributed neural system supports higher cognition, we need to consider how these domaingeneral areas intersect in the service of a range of different

Electronic supplementary material The online version of this article (https://doi.org/10.1007/s00429-018-1716-z) contains supplementary material, which is available to authorized users.

Anna M. Woollams

anna.woollams@manchester.ac.uk

1 Neuroscience and Aphasia Research Unit, School of Biological Sciences, Zochonis Building, University of Manchester, Brunswick Street, Manchester M13 9PL, England, UK tasks. Current methods based on subtraction logic, however, tend to focus on revealing dissociated, task-specific subcomponents. We outline here a new multi-dimensional correlation-based approach that allows the identification of the primary domain-general cognitive components that underpin a range of tasks, as well as their neural correlates. We apply this generalizable approach to the test case of reading, because it is a domain where the idea of shared processing across language activities has been explicitly formulated and computationally implemented as the primary systems hypothesis (Seidenberg and McClelland 1989; Plaut et al. 1996; Patterson and Lambon Ralph 1999).

Reading is a fundamental human capacity that is supported by a distributed network of neural regions. Although most adults are able to read fluently with little effort, it is, nevertheless, a late-acquired ability both phylogenetically and ontogenetically. Reading therefore builds upon the foundations of more basic, long-established neurocognitive functions. This idea forms the basis for the primary systems account of acquired reading disorders (Patterson and Lambon Ralph 1999), which proposes that different types 
of acquired dyslexia result from disruption to phonological, semantic, or visual processing (Crisp and Lambon Ralph 2006; Woollams et al. 2007; Roberts et al. 2013). Previous investigations of acquired dyslexia have supported this account by focussing on the relationship between reading and primary systems functions at the behavioural level. For the first time, this study simultaneously explored both neural and behavioural correlates through the application of sophisticated multi-dimensional neuropsychological and lesion-symptom mapping approaches to data collected from a large case-series of chronic post-stroke aphasic patients. These patients have persistent problems in understanding and/or producing speech due to deficits in phonological and/ or semantic processing (Lambon Ralph et al. 2002; Butler et al. 2014). If the primary systems account is correct, then there should be a strong convergence and triangulation between reading performance, primary cognitive systems, and their neural bases.

Functional neuroimaging of healthy participants has implicated a dorsal phonologically-related 'direct' pathway and a ventral semantically-mediated pathway in reading (Cattinelli et al. 2013; Taylor et al. 2013; Hoffman et al. 2015a), yet these data do not speak to the necessity of the regions involved. Lesion data from patients provide unique insights into the cognitive and neural bases of reading (Woollams 2014). In one of the first case-series studies to directly assess the primary systems account of reading deficits, Crisp and Lambon Ralph (2006) recruited 12 stroke aphasic patients on the basis of their reading behaviour, namely the presence of phonological-deep dyslexic symptoms (enhanced lexicality effects due to poor reading of nonwords, imageability effects, or semantic errors in reading). As predicted by the primary systems account, patients' nonword reading was related to phonological processing ability, as measured by phoneme manipulation performance. In addition, the advantage for words over nonwords (the lexicality effect) in reading was related to semantic processing ability, as measured by synonym judgement performance. Hence, word reading performance benefitted from residual semantic processing. The advantage for concrete over abstract words in reading was larger for patients with poor phoneme manipulation and poor synonym judgement, indicating that the processing of difficult abstract words draws on both semantic and phonological processing capacities. Although this study provided strong behavioural evidence to support the primary systems account, it did not consider how these effects related to the location of the patients' brain lesions.

Ripamonti et al. (2014) identified 33 cases of phonological dyslexia amongst 59 individuals recruited for post-stroke reading problems in the subacute stage. Using a lesion overlap approach, they compared phonological dyslexia (defined as better reading of concrete nouns than nonwords and a low incidence of stress assignment errors) over undifferentiated dyslexia (defined as better reading of concrete nouns than nonwords and a high incidence of stress assignment errors or equivalent reading accuracy for concrete nouns than nonwords with a low incidence of stress assignment errors). Areas of damage specific to phonological dyslexia consisted of the left posterior and superior insula and pars opercularis, with an additional VLSM analysis of nonword reading accuracy identifying these areas plus a broader network of perisylvian regions, including the pars triangularis, anterior superior temporal gyrus, temporal pole, middle frontal gyrus, and post-central gyrus. While this study did highlight lesion sites associated with nonword reading deficits, it was not able to directly test the primary systems account, because phonological processing was only minimally assessed by word and nonword repetition, and the relationship of these scores to either reading accuracy or neural integrity was not explored.

Fiez et al. (2006) took a lesion-based approach to participant selection in their study of the neural correlates of reading. They selected 11 patients with circumscribed lesions to the left frontal operculum on the basis of the consistent activation of this region in studies of healthy individuals when reading nonwords. As expected according to the primary systems account, these patients showed relatively greater deficits for nonwords than words as compared to both brain damaged and matched healthy control groups. They also had difficulty in reading aloud low-frequency words with inconsistent spelling-sound correspondences, and, indeed, stronger frontal opercular activation has been observed for these items relative to other words in imaging studies of healthy readers. Fiez et al. found deficits for their patients on a variety of phonological tasks (verbal working memory and phonological discrimination, pseudohomophone and rhyme discrimination). These results show a strong association between frontal opercular damage and reading ability, and between frontal opercular damage and phonological processing, although the relationship between phonological and reading abilities was not directly assessed. While the results are highly consistent with a primary systems view, the focus on patients with frontal opercular lesions necessarily limits coverage of the broader left hemisphere reading network. Hence we cannot know from this study if there are other areas that are particularly important specifically for nonword or word reading.

A more comprehensive lesion-based approach was adopted by Rapcsak et al. (2009), who recruited 31 patients on the basis of the presence of lesions involving one or more of five left perisylvian regions, as identified by their consistent activation in phonological processing tasks in functional neuroimaging meta-analyses (Vigneau et al. 2006). Performance for nonwords was significantly worse than for words, and the majority of patients, therefore, qualified as 
phonologically dyslexic. Patients were worse than controls at reading aloud nonwords, and also both regular and irregular words. As expected according to the primary systems account, reading performance for both nonwords and words was strongly related to phonological processing ability as measured over a range of receptive and expressive tests (repetition, rhyme processing, and phoneme processing). Neuroanatomically, damage to any of the five left perisylvian regions of interest corresponded to enhanced lexicality effects in reading, consistent with the involvement of a distributed phonological processing network in supporting nonword reading. What is not known from this study is which elements of this network were also necessary for phonological processing.

Most recently, Boukrina et al. (2015) studied the reading performance of 11 left hemisphere stroke patients undergoing rehabilitation in the subacute stage. Their reading aloud of words and nonwords was assessed, along with receptive tests of semantic, phonological, and orthographic processing, all of which involved a reading component. Behaviourally, a significant relationship was found between reading of high imageability, low frequency, low consistency words, and semantic task performance, and between reading of nonwords and phonological task performance, as would be expected according to the primary systems account. Using a lesion overlap approach, they were able to isolate areas specific to impairment on the phonological rhyme task over patients with no impairment on this task, and this corresponded to a wide network of frontal, parietal, and temporal left hemisphere regions. While this study identified the neural network supporting phonological processing, the neural correlates of reading aloud words or nonwords were not considered.

In summary, previous research has focussed on the association between primary systems abilities and reading performance in behaviour, on the neural correlates of reading performance or phonological processing separately, or on the role of specific brain regions in both abilities. The goal of this study was to provide the first large-scale quantitative assessment of the primary systems hypothesis simultaneously at both the behavioural and neural levels. This account predicts a strong overlap between reading performance and the status of each primary system, both behaviourally and neurally. To achieve this, we advanced on previous work in four ways. First, we considered the reading performance of 43 chronic poststroke aphasic patients selected purely because they experience persistent difficulties in spoken language processing (Lambon Ralph et al. 2002; Butler et al. 2014), rather than on the basis of their reading behaviour or lesion location (cf. Crisp and Lambon Ralph 2006; Fiez et al. 2006; Rapcsak et al. 2009; Ripamonti et al. 2014). This approach allows a test of the primary systems prediction that problems with spoken language should be accompanied by reading deficits. Second, our representative sample of chronic stroke aphasic patients gave sufficient variation in lesion location to allow a continuous rather than categorical approach to identifying the neural basis of reading deficits (cf. Ripamonti et al. 2014; Sebastian et al. 2014; Boukrina et al. 2015). Third, we were able to isolate and quantify each patient's primary systems capacities by using principal components analysis to distil optimal orthogonal measures of semantic and phonological ability from a large neuropsychological battery (Lambon Ralph et al. 2002, 2003). Finally, the uncorrelated nature of these phonological and semantic factors allowed us to identify the neural structures that uniquely correlate with these primary language abilities using voxel-based correlational methodology (see Butler et al. 2014), which treats both behavioural and neural measures as continuous variables. Accordingly, the specific targets of our study were to confirm the strong relationships between reading performance and the status of the primary systems and establish the intersection of their associated lesion maps.

\section{Materials and methods}

\section{Participants}

Forty-three chronic stroke patients (either ischaemic or haemorrhagic) were recruited, who had persistent impairments in producing and/or understanding spoken language. All patients were at least 12 months post-stroke at the time of scanning and assessment, and were native English speakers with normal or corrected-to-normal hearing and vision. Participants were excluded if they had any contraindications for scanning, were pre-morbidly left handed, had more than one stroke, or had any other significant neurological conditions. Informed consent was obtained from all participants prior to participation under approval from the local ethics committee. Data from a healthy age- and education-matched control group (8 female, 11 male) were used as the reference for identification of areas of neural abnormality.

\section{Neuropsychological assessments}

Assessments were conducted with participants over several testing sessions as required to complete the assessments. In addition to the BDAE (Goodglass and Kaplan 1983; Goodglass et al. 2000), a battery of language tests was administered to assess the participants' language and cognitive abilities. The language assessments included a variety of subtests from the Psycholinguistic Assessments of Language Processing in Aphasia (PALPA) battery (Kay et al. 1992), including: same-different auditory discrimination using nonword minimal pairs (PALPA 1); same-different 
auditory discrimination using word minimal pairs (PALPA 2); immediate repetition of nonwords (PALPA 8); delayed repetition of nonwords (PALPA 8); immediate repetition of words (PALPA 9); and delayed repetition of words (PALPA 9). A number of tests from the 64-item Cambridge Semantic Battery (Bozeat et al. 2000) were also included: the spoken word-to-picture matching task; a written word-to-picture matching version of the same task; the picture version of the Camel and Cactus Test; and the picture naming test. To increase sensitivity to mild naming deficits, the 60-item Boston Naming Test (BNT) (Kaplan et al. 1983) was also used. Similarly, to increase sensitivity to subtle semantic deficits, a 96-trial synonym judgement test with words presented in spoken and written form (Jefferies et al. 2009) was also used. To capture syntax-level deficits, the spoken sentence comprehension task from the Comprehensive Aphasia Test (CAT) (Swinburn et al. 2005) was administered. The additional cognitive tests included forward and backward digit span (Wechsler 1987); the Brixton Spatial Rule Anticipation Task (Burgess and Shallice 1997); and Raven's coloured progressive matrices (Raven 1962).

On language assessments, apart from the CAT sentence comprehension test (Swinburn et al. 2005), participants were scored on their first response. For the CAT test, two points are given for a correct response and one point is given for delayed correct responses or self-corrections. For the two naming assessments, participants' responses were marked correct if they were given within $5 \mathrm{~s}$ of presentation. Minor articulatory dysfluencies, but not phonological errors, in responses were accepted as correct. Repetition of auditory stimuli was provided if requested by participants.

Assessments of reading aloud were included in the same testing sessions as the neuropsychological background measures. We used two tests from the PALPA (Kay et al. 1992): the 30 item nonword syllable length list (PALPA 8) and the 80 -item imageability by frequency list (PALPA 31), which consists of 20 high-frequency concrete words, 20 low-frequency concrete words, 20 high-frequency abstract words, and 20 low-frequency abstract words. Normative data for the imageability by frequency list for 32 healthy control participants showed a lower bound for the hardest low-frequency abstract words of $98 \%$ correct $(\mathrm{SD}=0.34)$. In all reading tasks, the patients' first response was used for scoring purposes, and in the case of nonwords, any plausible pronunciation was considered correct. Scores for each word type for each patient are provided in Table 1.

\section{Behavioural data analysis}

Participants' scores on all assessments were entered into a principal components analysis (PCA) with varimax rotation (conducted with SPSS 16.0). Factors with an eigenvalue of 1.0 and above were extracted and then rotated. Following orthogonal rotation, the factor loadings of each test (presented in Table 2) allowed interpretation of what cognitivelanguage primary process was represented by that factor. Individual participants' scores on each extracted factor were then used as predictors of reading behaviour and as covariates in the neuroimaging analysis.

The scores from the principal components analysis for each factor are presented for each patient in Table 1. To assess the primary systems hypothesis at the behavioural level, these individual factor scores were correlated with reading accuracy for concrete and abstract words and nonwords using Pearson's correlations (conducted with SPSS 16.0), as we expected to see linear relationships (and these are the format of relationships assessed in the lesion-symptom mapping analyses). Effects were considered significant if their $p$ value fell below 0.05 .

\section{Acquisition of neuroimaging data}

High-resolution structural T1-weighted Magnetic Resonance Imaging (MRI) scans were acquired on a $3.0 \mathrm{~T}$ Philips Achieva scanner (Philips Healthcare, Best, The Netherlands) using an eight-element SENSE head coil for the first 31 patients and a 32-channel head coil for the remaining 12 patients. A T1-weighted inversion recovery sequence with $3 \mathrm{D}$ acquisition was employed, with the following parameters: TR (repetition time) $=9.0 \mathrm{~ms}$, TE (echo time) $=3.93 \mathrm{~ms}$, flip angle $=8^{\circ}, 150$ contiguous slices, slice thickness $=1 \mathrm{~mm}$, acquired voxel size $1.0 \times 1.0 \times 1.0$ $\mathrm{mm}^{3}$, matrix size $256 \times 256$, FOV $=256 \mathrm{~mm}$, TI (inversion time $)=1150 \mathrm{~ms}$, SENSE acceleration factor 2.5 , total scan acquisition time $=575 \mathrm{~s}$.

\section{Neuroimaging data analysis}

Each participant then had an MRI scan within a few weeks of completion of the behavioural assessments. Structural MRI scans were preprocessed with Statistical Parametric Mapping software (SPM8: Wellcome Trust Centre for Neuroimaging, http://www.fil.ion.ucl.ac.uk/spm/). The images were normalised into standard Montreal Neurological Institute (MNI) space using a modified unified segmentation-normalisation procedure optimised for focal lesioned brains (Seghier et al. 2008). Although referred to as an automated 'lesion' segmentation method, the technique detects areas of unexpected tissue class and, therefore, identifies diminished grey and white matter and increased CSF. This method has been shown to perform at an acceptable level relative to the gold standard of cost-function masking with a hand-traced lesion mask (Wilke et al. 2011), particularly in the case of large lesions, as seen in the majority of patients in our sample. Data from all participants with stroke aphasia and all healthy controls were entered into the 


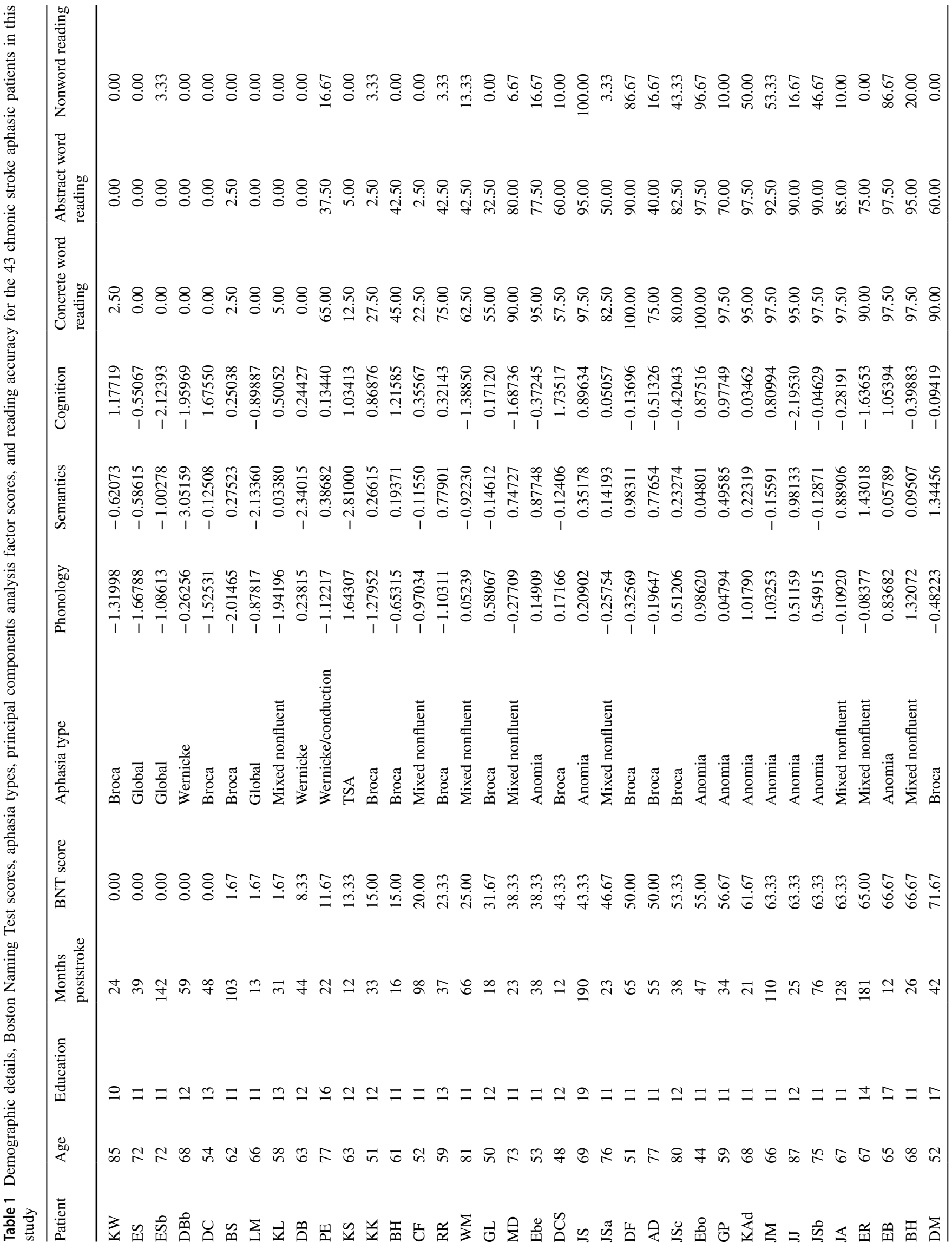




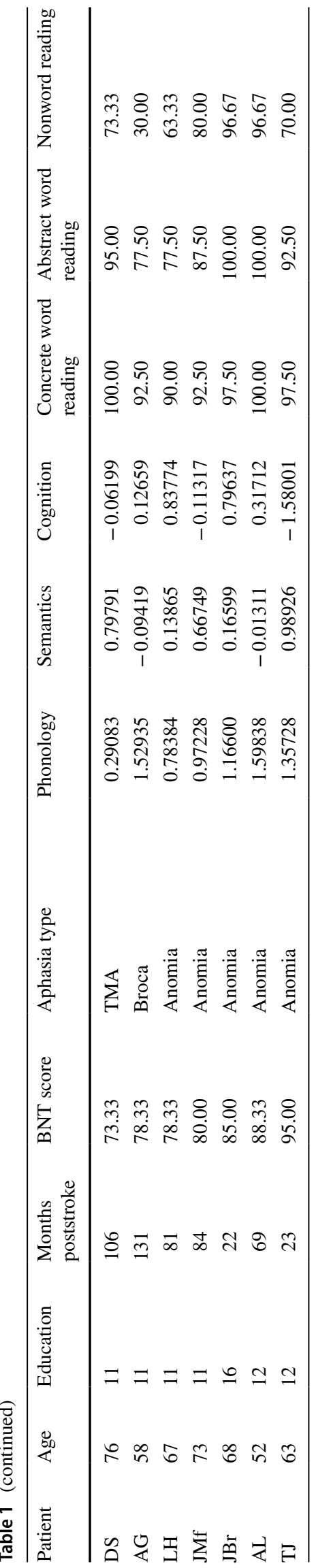

Table 2 Factor loadings for each test on the rotated factors identified in the principal components analysis

\begin{tabular}{llll}
\hline Test & Phonology & Semantics & Cognition \\
\hline Immediate nonword repetition & $\mathbf{0 . 8 8}$ & 0.17 & 0.15 \\
Delayed nonword repetition & $\mathbf{0 . 9 1}$ & 0.06 & 0.18 \\
Immediate word repetition & $\mathbf{0 . 8 6}$ & 0.25 & 0.11 \\
Delayed word repetition & $\mathbf{0 . 8 8}$ & 0.30 & 0.13 \\
Cambridge picture naming & $\mathbf{0 . 7 5}$ & $\mathbf{0 . 6 0}$ & 0.06 \\
Boston picture naming & $\mathbf{0 . 7 6}$ & $\mathbf{0 . 5 5}$ & -0.04 \\
Minimal pairs nonwords & 0.44 & 0.44 & $\mathbf{0 . 5 5}$ \\
Minimal pair words & $\mathbf{0 . 5 2}$ & $\mathbf{0 . 5 7}$ & 0.41 \\
Spoken word-to-picture match- & 0.20 & $\mathbf{0 . 8 6}$ & 0.27 \\
$\quad$ ing & & & \\
Written word-to-picture match- & 0.11 & $\mathbf{0 . 8 3}$ & 0.44 \\
ing & & & \\
CAT spoken sentence compre- & 0.59 & 0.29 & $\mathbf{0 . 5 7}$ \\
hension & & & \\
Synonym judgement test & 0.41 & $\mathbf{0 . 5 4}$ & 0.49 \\
Camel and cactus test pictures & 0.01 & 0.48 & $\mathbf{0 . 6 9}$ \\
Brixton spatial anticipation test & 0.31 & 0.23 & $\mathbf{0 . 6 7}$ \\
Ravens coloured progressive & 0.04 & 0.12 & $\mathbf{0 . 9 0}$ \\
matrices & & & \\
Digits forward & $\mathbf{0 . 8 3}$ & 0.09 & 0.23 \\
Digits backward & $\mathbf{0 . 7 2}$ & 0.06 & 0.38 \\
\hline
\end{tabular}

Loadings greater than 0.5 are highlighted in bold

segmentation-normalisation. Images were then smoothed with an $8 \mathrm{~mm}$ full-width-half-maximum (FWHM) Gaussian kernel. The lesion of each patient was automatically identified using an outlier detection algorithm, compared to healthy controls, based on fuzzy clustering. The default parameters were used apart from the lesion definition 'U-threshold', which was set to 0.5 to create a binary lesion image. The images generated were used to create the lesion overlap presented in Fig. 1.

Brain regions where tissue concentration (as represented by the continuous values of the abnormality map from the unified segmentation-normalisation procedure) were related to behavioural measures were identified using voxel-based correlational methodology (VBCM) (Tyler et al. 2005), a variant of voxel-based lesion-symptom mapping (VSLM) (Bates et al. 2003) in which both the behaviour and tissue concentration measures are treated as continuous variables (conducted in SPM8 using voxel-based morphometry). We first identified the key dimensions underpinning performance in this sample of stroke aphasic patients on our neuropsychological battery by entering the phonology, semantics, and cognitive factors simultaneously using VBM in SPM8. We then conducted independent analyses (due to the intercorrelation between reading measures) of reading accuracy for concrete and abstract words and nonwords. The degree of overlap of each of these reading maps with the PCA factor 


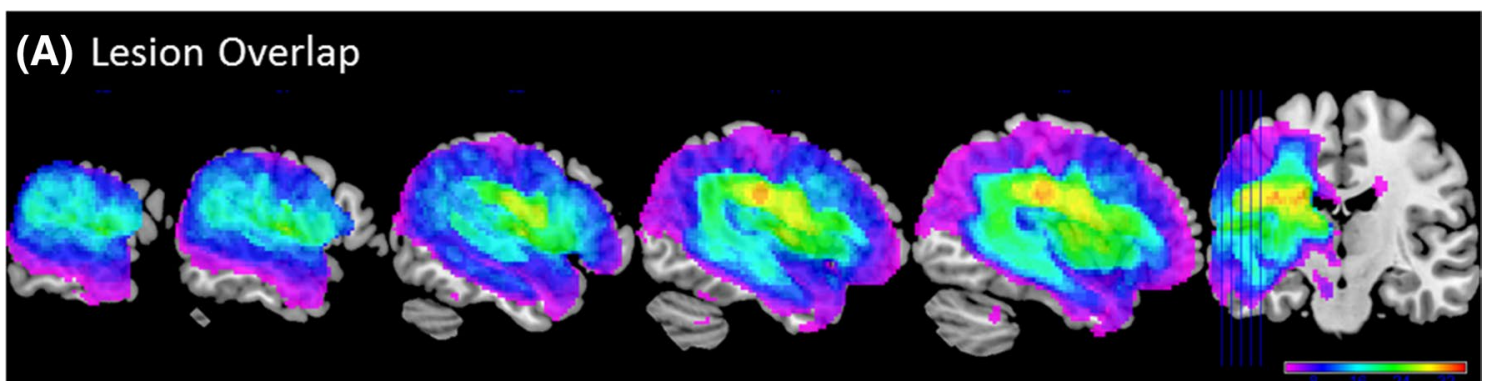

(B) Phonology \& Semantics

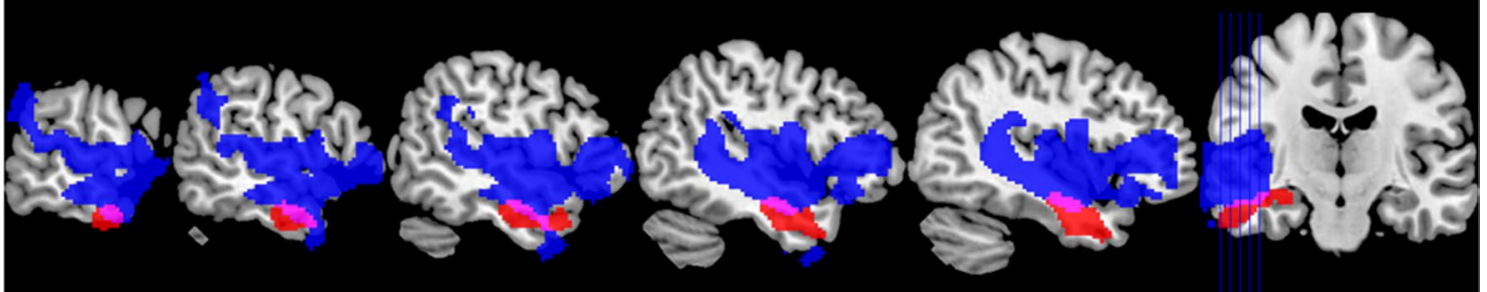

(C) Concrete Words, Abstract Words, \& Nonwords

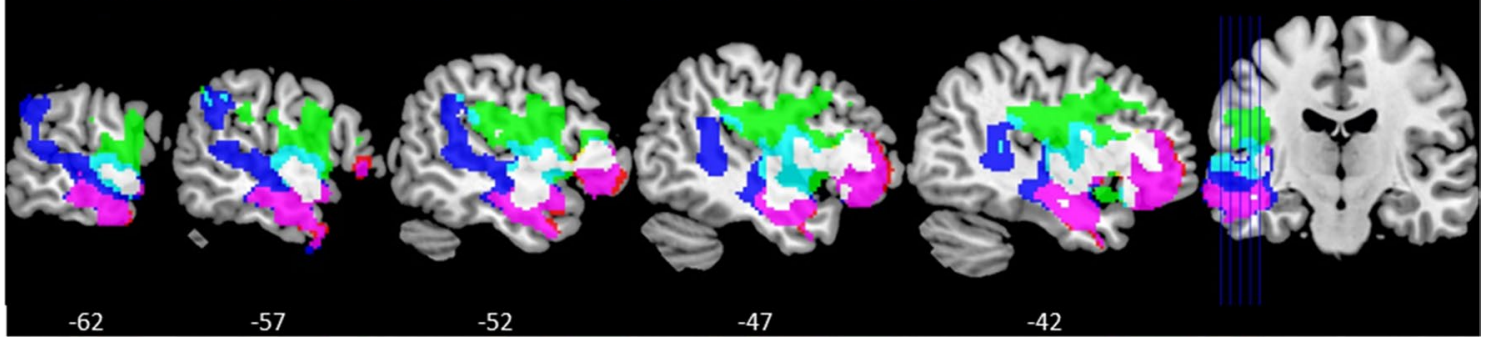

Fig. 1 a Lesion overlap map across the 43 patients (threshold 3-35); b regions found to relate significantly and uniquely to phonological (blue) and semantic (red) factors in simultaneous VBCM analyses, with the overlap shown in violet. c Regions found to relate significantly to nonwords (green), abstract words (blue), and concrete words (red), with overlap of nonwords and abstract words in cyan, of abstract and concrete words in violet, and of all three in white. Overlap between maps represent a conjunction as recommended by Nichols et al. (2005) with a significance level that is the product of that of each map (i.e., $p<0.000025$, voxel level; $p<0.0025$, FWEcorrected). Overlays show areas significant at $p<0.005$ voxel level, $p<0.05$ FWE-corrected cluster level, image threshold $(t)=2.7$

lesions within the semantic cluster in this predominantly phonologically impaired stroke aphasic sample.

\section{Results}

\section{Behavioural analyses}

Across the battery of background neuropsychological tests, these patients had marked and varied deficits. A varimaxrotated principal components analysis (PCA) produced a three-factor solution which accounted for $79 \%$ of variance in participants' performance ( $\mathrm{F} 1=58 \%$; $\mathrm{F} 2=14 \%, \mathrm{~F} 3=7 \%)$. The factor loadings of each of the different behavioural assessments are given in Table 2. Tasks which tapped input and/or output phonology (e.g., repetition, picture naming, and digit span) loaded heavily on the first factor, which we term 'phonology'. Tasks involving conceptual processing (e.g., picture naming, word-to-picture matching, and nature of permutation testing and the lower incidence of 
synonym judgement) loaded heavily on the second factor, which we term 'semantics'. The assessments that loaded heavily on the third factor involved reasoning (e.g., Brixton, Raven's, Camel, and Cactus) and we, therefore, labelled this factor 'cognitive'.

Correlations between the scores from the principal components analysis and reading accuracy are presented in Table 3. As expected according to the primary systems hypothesis: reading of concrete words correlated significantly with phonology and slightly more strongly with semantics, whereas reading of abstract words correlated significantly with both semantics and more strongly with phonology; accuracy of nonword reading correlated only with phonology. Unsurprisingly given the automatic nature of adult performance, no reading measure correlated significantly with the cognitive factor, and hence, it will not be considered further.

A series of repeated-measures ANCOVAs were conducted in SPSS 16.0 to assess whether there were significant differences between the predictive capacity of each of the PCA factors over different word types. These involved the PCA factors as predictors (either phonology or semantics) and word type as a within-participant variable (nonwords vs abstract words or abstract vs concrete words), yielding four separate analyses. Phonology predicted performance for nonwords and abstract words to a similar degree $[F(1$, $41)=2.84, p=0.100]$ and also abstract and concrete words to a similar degree $[F(1,41)=1.34, p=0.254]$. Semantics predicted performance for nonwords significantly less well than for abstract words $[F(1,41)=12.08, p=0.001]$, and performance for concrete words significantly better than abstract words $[F(1,41)=5.57, p=0.023]$. Overall, then, the behavioural results show that phonology is an important predictor for all word classes, but there is a graded relationship with semantics which is weakest for nonwords and strongest for concrete words.

\section{Neuroimaging analyses}

A lesion overlap map for stroke aphasic participants is provided in Fig. 1a (range 3-35 patients). As would be expected, this primarily covers the left hemisphere area supplied by the middle cerebral artery (Phan et al. 2005). The maximum

Table 3 Correlations between the principal components analysis factors and reading measures

\begin{tabular}{llll}
\hline Measure & Phonology factor & Semantics factor & Cognitive factor \\
\hline Concrete & $\mathbf{0 . 6 5 1}$ & 0.670 & -0.038 \\
Abstract & $\mathbf{0 . 7 1 4}$ & $\mathbf{0 . 5 8 2}$ & -0.029 \\
Nonwords & $\mathbf{0 . 5 9 5}$ & 0.270 & 0.172 \\
\hline
\end{tabular}

Bolded values are significant at $p<0.0005$ number of participants who had a lesion in any one voxel was 35 (in the central operculum and anterior arcuate). Anatomical labels are based on the Harvard-Oxford and NatBrainLab templates provided with MRICron (version 4).

The lesion map demonstrates that we had good coverage of key left hemisphere regions associated with spoken language processing. In voxel-based correlational methodology (VBCM), both voxel integrity and behavioural measures are treated as continuous; all observations are used in the analysis for each voxel over the whole brain. This contrasts with Voxel-Based Lesion-Symptom Mapping, in which voxel integrity is binarised to produce groups of intact vs lesioned patients. This approach is problematic when one group is very small (i.e., in voxels lesioned in very few patients), and hence, a minimum lesion cutoff is applied. We acknowledge that, in this sample, the distribution of voxel integrity values could be bi-modal; however, as previous VBCM studies have not applied a lesion cutoff (Tyler et al. 2005; Butler et al. 2014; Halai et al. 2017), we did not do so in the present study.

As can be seen in Figure S1, and in agreement with our previous work (Butler et al. 2014; Halai et al. 2017), lesion volume was correlated with a number of regions around the left perisylvian fissure, which is to be expected give this sample all had MCA infarcts (Phan et al. 2005). Although some researchers suggest that lesion volume should be entered as a covariate in lesion-symptom mapping analyses to control for global severity, we would argue that this approach is too conservative when the areas correlated with lesion volume overlap with the key functional areas of interest. This is certainly so in this study, as the inferior, posterior, and superior extensions of lesions involve areas that we expect to be involved in reading. Moreover, it is these areas farther from the perisylvian focus that are most likely to be reading specific, so to control for lesion volume in our analyses would, in fact, work against detecting such regions. This would bias the results in favour of confirming the primary systems prediction that there should be no areas associated with reading of particular stimulus types that do not overlap with the regions supporting spoken language processing. For this reason, we did not control for lesion volume in our main analyses, but we do provide the results controlled for lesion volume controlled in Table S1. It must be kept in mind that the significant results within areas correlated with lesion volume must be interpreted with caution with respect to the specificity of their involvement in a particular behaviour.

Localising primary systems The VBCM results for the phonological and semantic factors are shown in Fig. $1 \mathrm{~b}$ and Table 4. Each map shows where tissue concentration covaries uniquely with a given factor score. All results are thresholded at $p<0.005$ voxel level, $p \leq 0.05$ family wise error (FWE)-corrected cluster level. 
Table 4 Results of the wholebrain VBCM analyses for speech measures

\begin{tabular}{|c|c|c|c|c|c|c|c|}
\hline Contrast & Cluster FWE & $k$ & $T$ & $x$ & $y$ & $z$ & Anatomy \\
\hline \multirow[t]{13}{*}{ Phonology } & \multirow[t]{13}{*}{$<0.0005$} & \multirow[t]{13}{*}{11,602} & 5.98 & -44 & -10 & 2 & Insular \\
\hline & & & 5.50 & -70 & -22 & -12 & Middle temporal gyrus pos \\
\hline & & & 4.47 & -58 & 16 & -8 & Temporal pole \\
\hline & & & 4.28 & -62 & -54 & 34 & Middle temporal gyrus temocc \\
\hline & & & 4.26 & -60 & -4 & -24 & Middle temporal gyrus ant \\
\hline & & & 4.19 & -56 & 28 & 0 & Inferior frontal gyrus $\mathrm{p}$ tri \\
\hline & & & 4.17 & -52 & 24 & -12 & Frontal orbital cortex \\
\hline & & & 4.02 & -62 & 6 & 2 & Precentral gyrus \\
\hline & & & 4.01 & -46 & 22 & 4 & Inferior frontal gyrus $\mathrm{p}$ tri \\
\hline & & & 3.96 & -48 & 32 & 8 & Inferior frontal gyrus $\mathrm{p}$ tri \\
\hline & & & 3.92 & -42 & -42 & 10 & Superior longitudinal fasciculus \\
\hline & & & 3.91 & -42 & 44 & 12 & Frontal pole \\
\hline & & & 3.77 & -68 & -48 & 16 & Supramarginal gyrus pos \\
\hline \multirow[t]{3}{*}{ Semantics } & \multirow[t]{3}{*}{0.003} & \multirow[t]{3}{*}{1456} & 4.93 & -56 & -8 & -26 & Middle temporal gyrus ant \\
\hline & & & 4.73 & -40 & -6 & -26 & Inferior longitudinal fas \\
\hline & & & 3.38 & -28 & -18 & -14 & Hippocampus \\
\hline \multirow[t]{26}{*}{ Fluency } & \multirow[t]{14}{*}{$<0.0005$} & \multirow[t]{14}{*}{5711} & 5.04 & -58 & 2 & 34 & Precentral gyrus \\
\hline & & & 4.75 & -60 & -2 & 8 & Precentral gyrus \\
\hline & & & 4.74 & -62 & 4 & 22 & Precentral gyrus \\
\hline & & & 4.26 & -44 & 10 & 50 & Middle frontal gyrus \\
\hline & & & 3.97 & -20 & 8 & 52 & Superior frontal gyrus \\
\hline & & & 3.62 & -42 & -8 & 60 & Precentral gyrus \\
\hline & & & 3.31 & -24 & -8 & 32 & Corticospinal tract \\
\hline & & & 3.18 & -30 & -4 & 22 & Putamen \\
\hline & & & 2.98 & -46 & 18 & 0 & Frontal operculum cortex \\
\hline & & & 2.96 & -32 & -12 & 66 & Precentral gyrus \\
\hline & & & 2.96 & -26 & -16 & 56 & Precentral gyrus \\
\hline & & & 2.91 & -20 & -10 & 48 & Precentral gyrus \\
\hline & & & 2.80 & -6 & -2 & 36 & Cingulum cingulate \\
\hline & & & 2.79 & -12 & 18 & 30 & Cingulate gyrus ant \\
\hline & \multirow[t]{12}{*}{0.021} & \multirow[t]{12}{*}{999} & 3.72 & -14 & 44 & 20 & Paracingulate gyrus \\
\hline & & & 3.40 & -16 & 52 & -10 & Inferior frontal occipital fas \\
\hline & & & 3.35 & -18 & 40 & 32 & Frontal pole \\
\hline & & & 3.33 & -46 & 48 & 4 & Frontal pole \\
\hline & & & 3.33 & -22 & 26 & 38 & Superior frontal gyrus \\
\hline & & & 3.29 & -30 & 46 & -16 & Frontal pole \\
\hline & & & 3.29 & -14 & 50 & 6 & Paracingulate gyrus \\
\hline & & & 3.22 & -24 & 54 & -6 & Frontal pole \\
\hline & & & 3.11 & -38 & 54 & -4 & Frontal pole \\
\hline & & & 3.06 & -36 & 38 & -20 & Frontal pole \\
\hline & & & 3.00 & -26 & 50 & 10 & Frontal pole \\
\hline & & & 2.77 & -20 & 44 & -16 & Frontal pole \\
\hline
\end{tabular}

All peaks fall within the left hemisphere
Performance on the phonological factor was uniquely correlated with a large cluster of a number of left hemisphere regions: frontal pole, middle frontal gyrus (MFG), inferior frontal gyrus (IFG) (triangularis and orbitalis), frontal and central opercular cortex, posterior insular cortex, temporal pole, planum polare, Heschl's gyrus, planum temporale, superior temporal gyrus (STG), middle temporal gyrus (MTG), parietal opercular cortex, and posterior supramarginal gyrus (SMG). The phonological cluster, therefore, overlapped with the anterior and posterior segments of the arcuate fasciculus, a key aspect of the dorsal language pathway (Wise 2003; Catani and ffytche 2005; Catani et al. 2005; 
Table 5 Results of the wholebrain VBCM analyses for reading measures

\begin{tabular}{|c|c|c|c|c|c|c|c|}
\hline Contrast & Cluster FWE & $k$ & $T$ & $x$ & $y$ & $z$ & Anatomy \\
\hline \multirow[t]{15}{*}{ Concrete words } & \multirow[t]{15}{*}{$<0.0005$} & \multirow[t]{15}{*}{7601} & 5.05 & -52 & 28 & 2 & Inferior frontal gyrus $\mathrm{p}$ tri \\
\hline & & & 4.60 & -58 & -6 & -24 & Middle temporal gyrus ant \\
\hline & & & 4.57 & -44 & -8 & -18 & Inferior longitudinal fas \\
\hline & & & 4.43 & -40 & 44 & -2 & Frontal pole \\
\hline & & & 4.42 & -40 & 36 & -14 & Frontal pole \\
\hline & & & 4.32 & -66 & -22 & -12 & Middle temporal gyrus pos \\
\hline & & & 4.25 & -42 & 34 & 10 & Inferior frontal gyrus $\mathrm{p}$ tri \\
\hline & & & 4.12 & -40 & 28 & -14 & Frontal orbital cortex \\
\hline & & & 3.94 & -34 & -4 & -30 & Temporal fusiform cortex ant \\
\hline & & & 3.72 & -60 & 2 & -8 & Superior temporal gyrus ant \\
\hline & & & 3.68 & -34 & 8 & -18 & Insular \\
\hline & & & 3.43 & -26 & 50 & 16 & Frontal pole \\
\hline & & & 3.38 & -42 & -12 & 4 & Insular \\
\hline & & & 3.23 & -40 & 10 & 4 & Frontal operculum cortex \\
\hline & & & 3.10 & -36 & -6 & 6 & Insular \\
\hline \multirow[t]{16}{*}{ Abstract words } & \multirow[t]{16}{*}{$<0.0005$} & \multirow[t]{16}{*}{10,845} & 5.32 & -44 & -8 & -18 & Inferior longitudinal fas \\
\hline & & & 4.68 & -52 & 28 & 2 & Inferior frontal gyrus $\mathrm{p}$ tri \\
\hline & & & 4.65 & -68 & -22 & -12 & Middle temporal gyrus pos \\
\hline & & & 4.52 & -42 & -12 & 2 & Insular \\
\hline & & & 4.40 & -40 & 36 & -14 & Frontal pole \\
\hline & & & 4.37 & -56 & 2 & -10 & Superior temporal gyrus ant \\
\hline & & & 4.36 & -58 & -6 & -24 & Middle temporal gyrus ant \\
\hline & & & 4.31 & -42 & 34 & 12 & Inferior frontal gyrus $\mathrm{p}$ tri \\
\hline & & & 4.19 & -40 & 26 & -16 & Frontal orbital cortex \\
\hline & & & 4.06 & -34 & -4 & -30 & Temporal fusiform cortex ant \\
\hline & & & 3.90 & -38 & 44 & -4 & Frontal pole \\
\hline & & & 3.68 & -54 & 2 & 4 & Central operculum cortex \\
\hline & & & 3.67 & -58 & -12 & 0 & Superior temporal gyrus ant \\
\hline & & & 3.62 & -34 & 8 & -18 & Insular \\
\hline & & & 3.53 & -58 & -48 & 32 & Supramarginal gyrus pos \\
\hline & & & 3.47 & -46 & -42 & 6 & Superior temporal gyrus pos \\
\hline \multirow[t]{15}{*}{ Nonwords } & \multirow[t]{15}{*}{$<0.0005$} & \multirow[t]{15}{*}{7221} & 5.34 & -56 & 2 & 6 & Central operculum cortex \\
\hline & & & 4.79 & -44 & -10 & 4 & Insular \\
\hline & & & 3.92 & -58 & 4 & 26 & Precentral gyrus \\
\hline & & & 3.76 & -46 & 18 & 2 & Frontal operculum cortex \\
\hline & & & 3.75 & -42 & 32 & 14 & Inferior frontal gyrus $\mathrm{p}$ tri \\
\hline & & & 3.72 & -38 & 6 & 34 & Middle frontal gyrus \\
\hline & & & 3.71 & -62 & 0 & -8 & Superior temporal gyrus ant \\
\hline & & & 3.62 & -64 & -10 & -2 & Superior temporal gyrus pos \\
\hline & & & 3.55 & -52 & 28 & 16 & Inferior frontal gyrus $\mathrm{p}$ tri \\
\hline & & & 3.54 & -46 & -28 & 26 & Superior longitudinal fasciculus \\
\hline & & & 3.53 & -30 & -30 & 18 & Planum temporale \\
\hline & & & 3.51 & -46 & -18 & 16 & Central operculum cortex \\
\hline & & & 3.42 & -48 & -8 & -16 & Superior temporal gyrus ant \\
\hline & & & 3.25 & -42 & 12 & -12 & Frontal operculum cortex \\
\hline & & & 3.11 & -38 & 24 & -18 & Frontal orbital cortex \\
\hline
\end{tabular}

All peaks fall within the left hemisphere 
Duffau et al. 2005; Parker et al. 2005; Saur et al. 2008) as well as much of the inferior longitudinal fasciculus, part of the ventral language pathway (Wise 2003; Parker et al. 2005; Catani and Mesulam 2008; Saur et al. 2008; Schmahmann and Pandya 2008; Duffau et al. 2009).

Performance on the semantic factor was uniquely related to a cluster of voxels centred on the white matter in the left anterior temporal lobe (ATL), extending to the temporal pole, planum polare, anterior and posterior MTG, and inferior temporal gyrus (ITG), and the anterior fusiform. The semantic cluster involved the anterior section of the inferior longitudinal fasciculus (ILF), the inferior aspect of the anterior commissure, and uncinate fasciculus, all of which comprise the ventral language pathway (Wise 2003; Parker et al. 2005; Catani and Mesulam 2008; Saur et al. 2008; Schmahmann and Pandya 2008; Duffau et al. 2009).

Mapping reading deficits The VBCM results for reading performance are shown in Fig. 1c and Table 5. There was a large area that was associated with reading accuracy for all three stimulus types. We refer to this as the core reading network, which spanned superior temporal and inferior frontal regions, including: frontal pole, middle frontal gyrus, frontal orbital cortex, frontal and central opercular cortex, IFG (pars triangularis and pars opercularis), insular cortex, precentral gyrus, temporal pole, planum polare, anterior STG, and anterior MTG. In terms of white-matter connectivity, this region overlapped primarily with the ILF but also the uncinate fasciculus.

Both nonword and abstract word reading were associated with tissue concentration in: the planum temporale and polare, parietal and central opercular cortex, insular cortex, precentral gyrus, posterior STG, and anterior MTG, and overlapped the anterior, posterior, and long segments of the arcuate fasciculus. Abstract and concrete word reading were associated with the frontal pole, frontal orbital cortex, IFG pars triangularis, insular cortex, anterior parahippocampal and anterior fusiform cortices, anterior and posterior ITG, temporal pole, planum polare, and anterior and posterior MTG, overlapping with the uncinate fasciculus, anterior ILF and IFOF. There were no regions that were associated with both nonword and concrete word reading.

Nonword reading was specifically associated with tissue concentration in: MFG, IFG (pars opercularis and triangularis), precentral gyrus, insular cortex, central and parietal opercular cortex, and anterior MTG, and overlapped the anterior and long segments of the arcuate fasciculus, frontal aslant tract [as presented by Catani et al. (2013)] and edged onto the internal capsule. Abstract word reading was associated specifically with posterior and temporo-occipital MTG, posterior STG, planum temporale, and anterior and posterior SMG, and overlapped the posterior arcuate, posterior ILF, and posterior inferior fronto-occipital fasciculus (IFOF). Concrete word reading was associated specifically with only a few areas in the frontal pole and insular cortex, just overlapping the anterior IFOF.

In summary, areas specific to nonword reading focussed on the MFG, IFG, inferior precentral gyrus, and insular and opercular cortices, and overlapped the arcuate fasciculus/ frontal aslant tract, while those areas specific to concrete and abstract words centred on inferior frontal and temporal regions, mainly MTG but also involving the fusiform, and overlapping both the ILF and uncinate. Interestingly, areas supporting reading of concrete words fell almost entirely within those supporting abstract words, with the latter showing an additional correlation with more posterior MTG and parietal regions.

Testing the primary systems hypothesis Figure 2 shows the overlap between areas associated with phonological processing and reading of each word type, next to the correlations between scores on the phonological factor and reading accuracy. There is clearly a strong relationship between non-reading phonological skills and reading accuracy for all three string types at both the behavioural and neural levels. This is consistent with the primary systems view that phonology is involved in reading of all strings, but, for words, semantic processing would make an appreciable contribution. Indeed, as can be seen in comparison of Fig. 1b, c, the core reading network falls within the phonological but not the semantic processing region.

As shown in Fig. 2a, a large inferior portion of the areas involved in nonword reading overlapped with the phonology map. This included areas specifically associated with nonword reading (parietal, central, and frontal opercula, insula and pre- and post-central gyri, pars opercularis, and triangularis) and areas shared between nonword and abstract word reading (central operculum, precentral gyrus, insula, and planum temporale). As can be seen in Fig. 2b, the areas involved in abstract word reading were almost entirely contained within the phonology map. This involved areas specifically associated with abstract word reading (planum temporale and polare, insular cortex, parietal and central opercular cortex, posterior MTG, posterior STG, and SMG). In addition, as can be seen in Fig. 2c, areas shared between concrete and abstract word reading overlapped with the anterior and inferior aspects of the phonology cluster frontal and temporal pole, IFG (pars triangularis), frontal orbital and insular cortex, frontal and central opercular cortex, planum polare, Heschl's gyrus, anterior and posterior MTG, and anterior STG.

Figure 3 shows the overlap between areas associated with semantic processing and reading of each word type, along with the correlations between semantic factor scores and reading performance. The strength of the relationship between the semantic factor and reading accuracy for each string type mirrors the proportion of the reading map that overlaps with the semantics map: this is minimal for the 


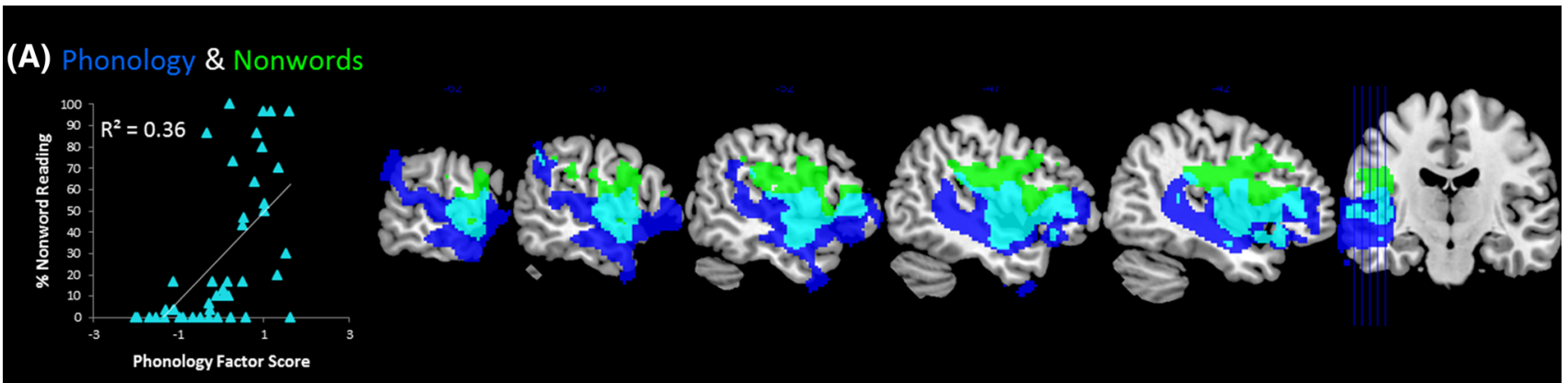

(B) Phonology \& Abstract Words
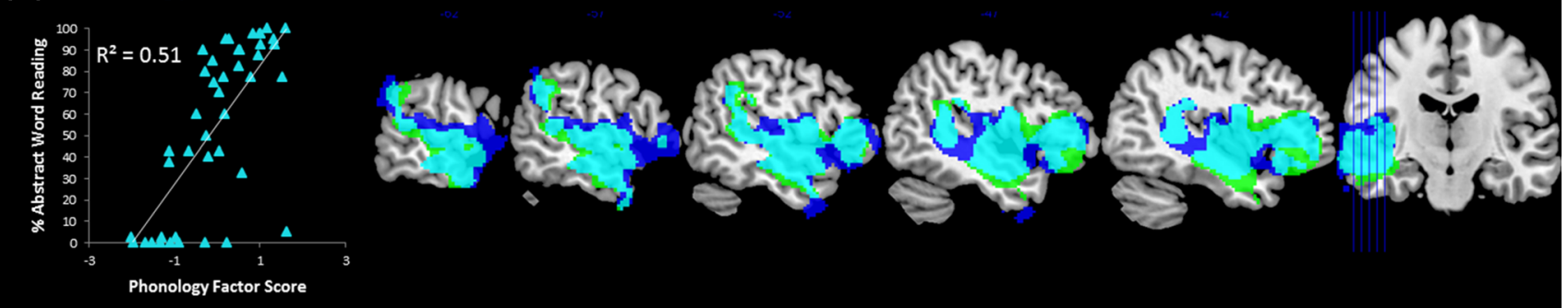

(C) Phonology \& Concrete Words
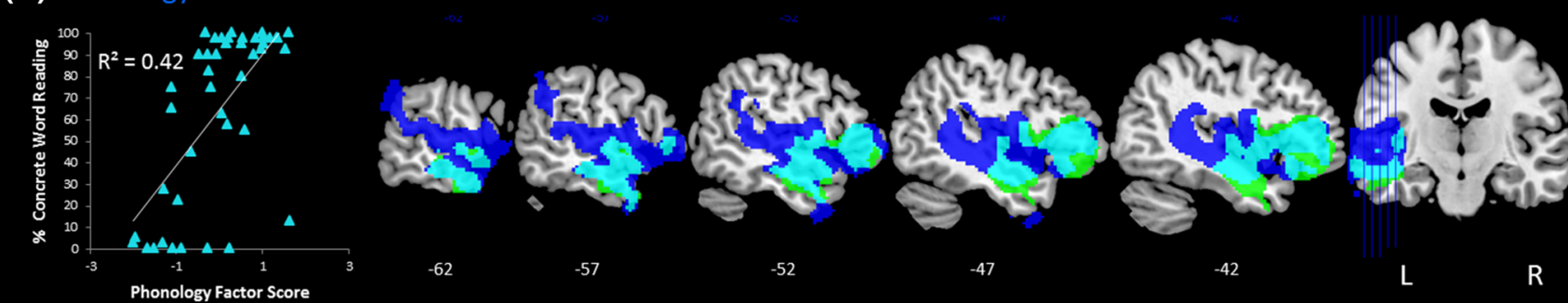

Fig. 2 Relationship between phonological processing and reading of: a nonwords, $\mathbf{b}$ abstract words, and $\mathbf{c}$ concrete words. Behavioural data are shown in the scatter plots on the left. VBCM results are shown on the right, with areas relating to the phonological factor in blue, areas related to reading performance in green, and the overlap between them in cyan. Overlays show areas significant at $p<0.005$ voxel level, $p<0.05$ FWE-corrected cluster level, image threshold $(t)=2.7$. Overlap between phonological processing and reading maps represents a conjunction as recommended by Nichols et al. (2005) with a significance level that is the product of that of each map (i.e., $p<0.000025$ voxel level, $p<0.0025$ FWE-corrected)

polare, and anterior and posterior MTG, overlapping with the uncinate fasciculus, anterior ILF, and anterior IFOF).

In addition to the reading areas identified that overlapped with primary systems, there were also some areas that did not. Most of these were small variations in the extent of the comparable lesion correlates. In the core reading network, a small portion of the pars opercularis that was implicated in reading all three types of strings fell outside the phonology cluster. Areas shared between nonwords and abstract words fell almost entirely within the phonology cluster, while those shared between the abstract and concrete words fell almost entirely within the phonology and semantics maps. The small areas associated specifically with concrete word reading (frontal pole and insular cortex, plus IFOF) did fall outside the primary systems regions, as did a few of the areas specifically associated with abstract word reading (posterior STG and particularly anterior and posterior SMG). 


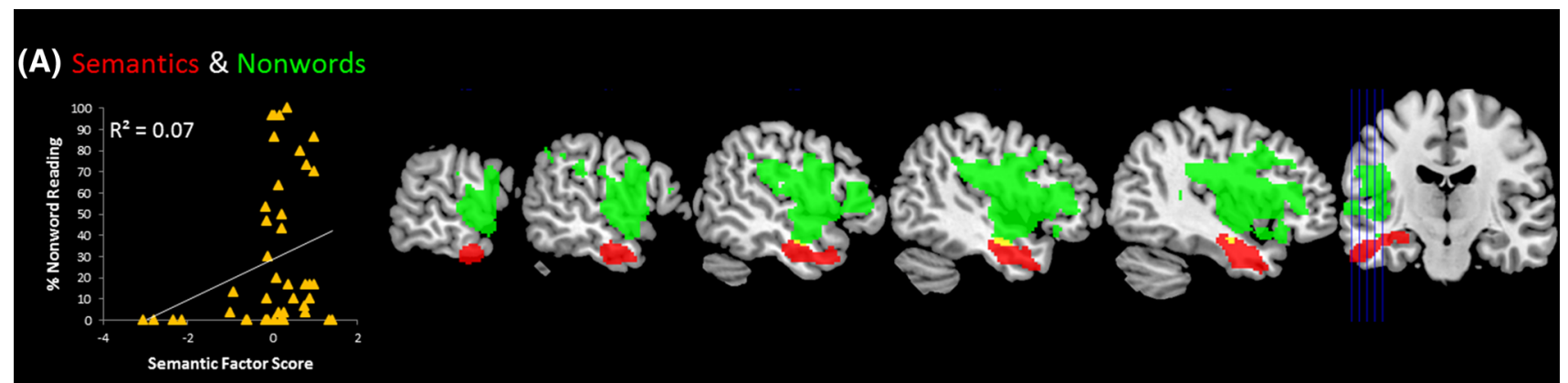

(B) Semantics \& Abstract Words

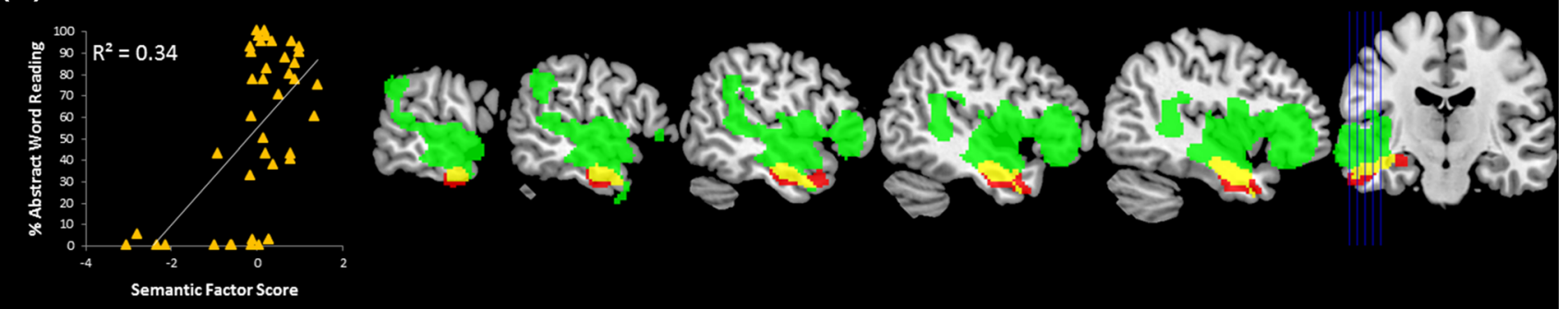

(C) Semantics \& Concrete Words

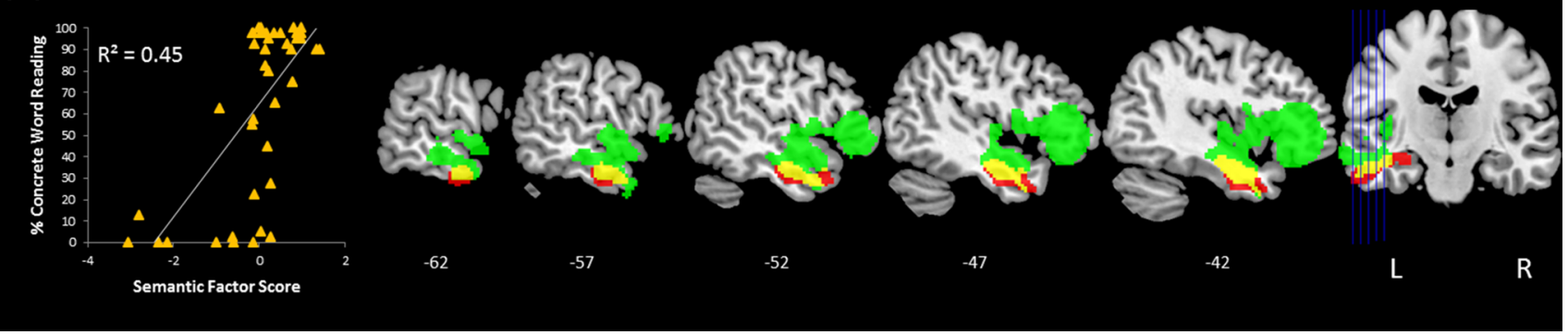

Fig. 3 Relationship between semantic processing and reading of: a nonwords, $\mathbf{b}$ abstract words, and $\mathbf{c}$ concrete words. Behavioural data are shown in the scatter plots on the left. VBCM results are shown on the right, with areas relating to the semantic factor in red, areas related to reading performance in green, and the overlap between them in yellow. Overlays show areas significant at $p<0.005$ voxel

Aside from these relatively small variations in the extents of the lesion correlates, there was one more notable and intriguing additional large superior frontal region associated specifically with nonword reading that did not overlap with the phonology map. This area included IFG pars opercularis and triangularis, middle frontal gyrus, pre- and particularly the post-central gyrus, plus central and parietal opercular cortex, and involved the anterior and long segments of the arcuate fasciculus, overlapping the inferior portion of the frontal aslant tract. This dorsal nonword reading-specific region which we observe has been previously shown to correlate with the control of motor speech output (Price 2012; Richardson et al. 2012) and with speech fluency/quanta (Catani et al. 2013; Basilakos et al. 2014)—which is a distinct underlying component of the aphasic multi-dimensional profile, statistically separate from phonology, semantics, and cognitive factors (Halai et al. 2017). level, $p<0.05$ FWE-corrected cluster level, image threshold $(t)=2.7$. Overlap between semantic processing and reading maps represents a conjunction as recommended by Nichols et al. (2005) with a significance level that is the product of that of each map (i.e., $p<0.000025$ voxel level, $p<0.0025$ FWE-corrected)

To isolate this additional primary speech motor output system, we mapped the lesion correlates of "words per minute" during description of the Cookie Theft picture (Goodglass et al. 2000), a commonly used index of speech fluency. As can be seen in Fig. 4, the behavioural Pearson's correlation between words per minute and reading performance, although significant for all word types (nonwords $r=0.553$, $p<0.0005$; abstract words $r=0.400, p=0.008$; concrete words $r=0.309, p=0.043$ ), is strongest for nonwords and weakest for concrete words. Moreover, there is considerable overlap between the maps of words per minute and nonword reading performance. Critically, this included many of the areas associated with nonword reading that did not overlap with the phonology factor, namely: the MFG, pre- and postcentral gyrus and IFG pars opercularis, and the anterior and long segments of the arcuate and the frontal aslant tract. In contrast, the overlap between words per minute and reading 


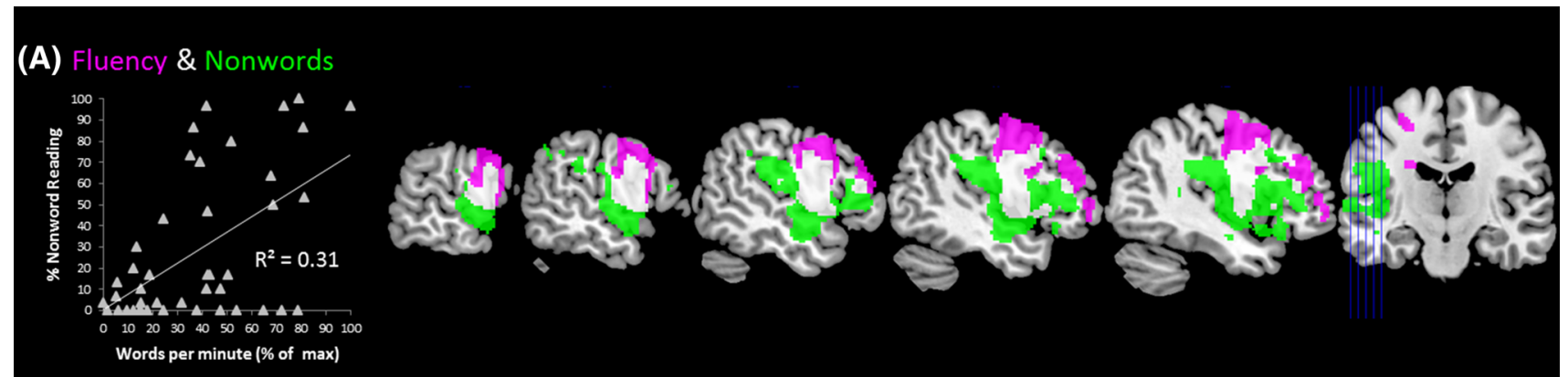

(B) Fluency \& Abstract Words
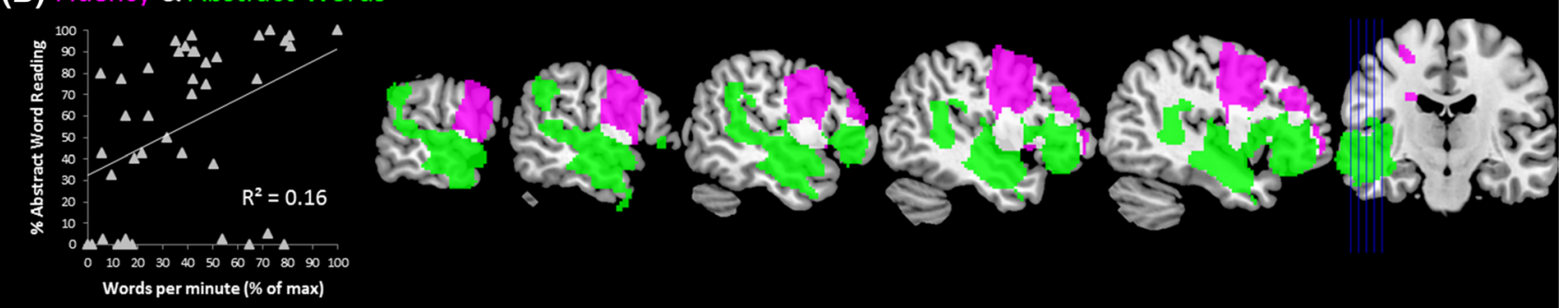

(C) Fluency \& Concrete Words
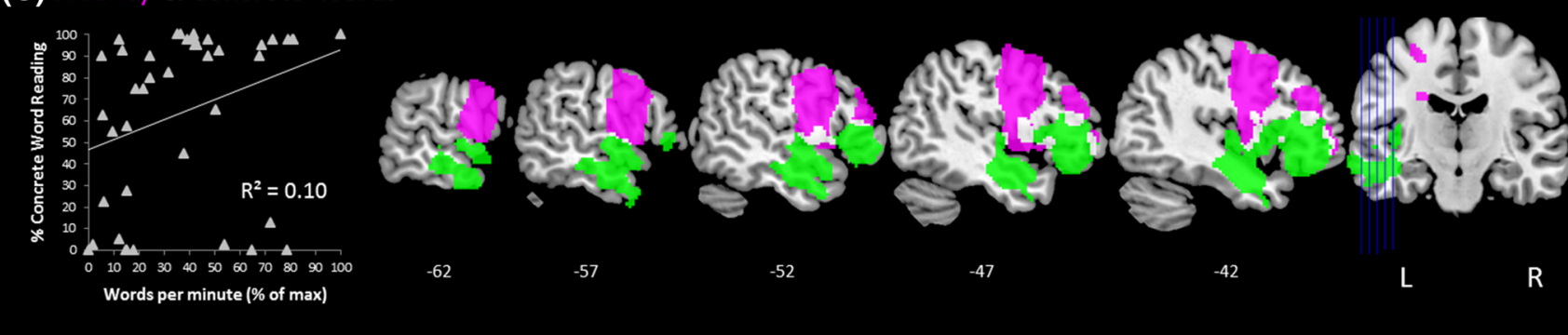

Fig. 4 Relationship between fluency and reading of: a nonwords, b abstract words, and $\mathbf{c}$ concrete words. Behavioural data are shown in the scatter plots on the left. VBCM results are shown on the right, with areas relating to fluency (as measured by words per minute in picture description as a percentage of the highest score) in violet, areas related to reading performance in green, and the overlap

performance for abstract words and concrete words fell almost entirely within the areas associated with phonology. Hence the lesion data indicate that there is a unique contribution of fluency to nonword reading over and above that of phonology. This then makes the very specific prediction that only nonword reading should show a significant relationship to fluency measures after controlling for phonological ability, and this was, indeed, the case in partial Pearson's correlations (nonwords $r=0.429, p=0.005$; abstract words $r=0.180, p=0.253$; concrete words $r=0.072, p=0.648$ ).

This result is theoretically significant for three reasons: it indicates that, by encompassing a large group of poststroke aphasic cases, we have been able to add an important additional component to the primary systems framework (phonology, semantics, vision, and controlled speech output); it demonstrates that nonword reading loads much more heavily on controlled speech output, presumably because the between them in white. Overlays show areas significant at $p<0.005$ voxel level, $p<0.05$ FWE-corrected cluster level, image threshold $(t)=2.7$. Overlap between fluency and reading maps represents a conjunction as recommended by Nichols et al. (2005) with a significance level that is the product of that of each map (i.e., $p<0.000025$ voxel level, $p<0.0025$ FWE-corrected)

pronunciation and articulation of nonwords involves a novel sequence; finally and relatedly, it suggests that patients with damage to this region will show nonword reading impairments disproportionate to those expected on the basis of their phonological scores alone.

\section{Discussion}

The purpose of this study was to provide a large-scale quantitative assessment of the primary systems account of acquired reading disorders, both behaviourally and neurally, for the first time. This neurocomputationally rooted theoretical framework predicts that the patterns of patients' reading deficits are systematically related to the status of more domain-general primary systems (Patterson and Lambon Ralph 1999). We isolated these primary systems using 
a principal components analysis of a large battery of neuropsychological data. Consistent with the primary systems hypothesis, we found that nonword reading correlated only with the phonological factor, and its lesion map clearly overlapped with the phonological neural cluster. Abstract word reading correlated with both phonological and semantic scores, and its lesion map overlapped with both the semantic and the phonological neural clusters. Concrete word reading also correlated with semantic and phonological scores, and its lesion correlate overlapped with the semantic and frontal and inferior aspects of the phonological cluster. In addition, our large-scale neuropsychological study allowed us to identify a novel additional primary systems component-prefrontal regions associated with controlled speech production-that are particularly important for nonword reading.

Our results demonstrate a superior-to-inferior gradation of reading specialisation across the dorsal and ventral pathways according to lexicality and concreteness. Given the typical perisylvian distribution of middle cerebral artery stroke lesions and its overlap with the phonological network, our results provide a unified explanation not only for the fact that phonological deficits are so prominent in poststroke aphasia (Schwartz et al. 2012; Butler et al. 2014), but also why nonword reading is so strongly undermined (Fiez et al. 2006; Rapcsak et al. 2009; Brookshire et al. 2014). Indeed, our study aligns with previous work concerning lesion sites associated with phonological dyslexia, which have implicated a variety of perisylvian regions, particularly the insula and LIFG, not only in post-stroke aphasia (Fiez et al. 2006; Rapcsak et al. 2009; Ripamonti et al. 2014) but also in primary progressive aphasia (Henry et al. 2012). Previous functional neuroimaging meta-analyses have found these areas to be consistently more active for nonword than word reading (Taylor et al. 2013) and reliably involved in phonological processing (Vigneau et al. 2006).

Performance for words is more robust not only through practice and experience (which, by definition, nonwords do not have), but also, because words can draw upon both semantics and phonological processing, as reflected by the overlap of word reading with both of these primary systems in our results. We observed a strong influence of concreteness on the extent to which word reading draws upon phonology and semantics. The semantic representations of abstract words are less rich than concrete words (Plaut and Shallice 1993; Paivio 2010) and, as a consequence, need to draw more heavily on phonological processing (Westbury and Moroschan 2009). Indeed, we found that abstract words overlapped with more of the phonological network than concrete words, encompassing areas in the planum temporale and polare, insula, parietal and central opercula, posterior MTG, and posterior STG and SMG. These results are in line with neuroimaging studies that have compared concrete and abstract words (Binder et al. 2005; Sabsevitz et al. 2005) and also with recent TMS and lesion mapping investigations that have implicated the SMG as involved in phonological processing specifically for words (Mirman and Graziano 2013; Pattamadilok et al. 2015; Sliwinska et al. 2015).

Both abstract and concrete words relied upon a ventral semantic pathway involving regions of the ATL, including the anterior fusiform, ITG, MTG, temporal pole, and underlying white-matter connections. This result converges with recent fMRI findings implicating the ventrolateral ATL in representing the meaning of both concrete and abstract words (Hoffman et al. 2015b). These anterior and inferior temporal semantic regions, implicated in reading of abstract and concrete words, are the same areas that have been associated with the deficits of exception word reading that define surface dyslexia (Ripamonti et al. 2014), which usually co-occurs with semantic dementia (Woollams et al. 2007, 2010; Wilson et al. 2009, 2012; Henry et al. 2012). Although this area can be challenging to image successfully in functional neuroimaging studies (Visser et al. 2010a), it can be with appropriate methods (Binney et al. 2010; Visser et al. 2010b). Such studies have found that, when healthy readers pronounce exception words, activation is observed in precisely the same ATL regions identified in the current study as supporting both semantics and word reading (Wilson et al. 2012; Hoffman et al. 2015a).

As predicted by the primary systems hypothesis, we observed strong associations between semantic and phonological aspects of speech processing and word and nonword reading. Our large-scale neuropsychological and lesionsymptom mapping study also allowed us to identify a new additional component for inclusion in the primary systems framework. Specifically, we found a large prefrontal cluster for nonword reading that fell outside the phonological and semantic neural-clusters. This additional region included the IFG pars opercularis and triangularis, middle frontal gyrus, pre- and post-central gyrus, plus central and parietal opercular cortex, and overlapped the anterior and long sections of the arcuate fasciculus and also the inferior portion of the frontal aslant tract.

This constellation of cortical and white-matter areas is implicated in aspects of preparation for and execution of speech (Price 2012; Dick et al. 2014). For patients with chronic stroke aphasia, damage to the IFG predicts motor speech impairments (Richardson et al. 2012) and integrity of both the anterior arcuate and aslant tract have been linked to speech fluency not only in stroke and progressive aphasia (Catani et al. 2013; Basilakos et al. 2014), but also in developmental speech production disorders (Kronfeld-Duenias et al. 2016) and neurosurgical stimulation studies (Kinoshita et al. 2015; Vassal et al. 2014; Fujii et al. 2015). Recently, fluency has been identified as a distinct factor that explains a significant variance in stroke aphasic performance, over and above phonology, semantics, and cognition (Halai et al. 
2017), with its lesion correlates encompassing the inferior portions of the frontal aslant tract.

The involvement of these speech-control areas and connections in nonword reading is to be expected, given that nonwords are by definition sequences of phonemes that would never have been pronounced before, and they will, therefore, draw most heavily on speech planning mechanisms. To directly establish the specific link between speech motor output and nonword reading, we considered the neural correlates of fluency in our sample, as measure by words spoken per minute. We found that there was clear overlap between the fluency maps and the areas specifically involved in nonword reading that fell outside the phonology maps. As predicted by the neural data, when we assessed the correlation between words per minute and reading performance controlling for phonological ability, it was only nonword reading that showed a significant relationship to fluency. Taken together, this pattern of results clearly indicates that the superior frontal cluster which we have identified is best seen as reflecting an additional primary motor output system that supports the speech production component of reading aloud.

The prefrontal/controlled-speech component which we have identified might explain why there have been a small number of reported individual cases with poor nonword reading yet better than expected phonological processing skills. If nonword reading is supported by phonology and speech motor output, then impairments of controlled speech output may not generate a large phonological impairment but would reduce nonword reading accuracy (e.g., Tree and Kay 2006). This is potentially theoretically significant as these kinds of cases have been cited as evidence against the primary systems account (Tree 2008). There is, however, more than one reason for poor nonword reading. Pure alexic patients (Cumming et al. 2006), who have reading and visual impairments consequent on lesions in the ventral occipitotemporal region (Roberts et al. 2013), show a nonword reading impairment not due to any phonological impairment, but, rather, because their impaired recognition of words is ameliorated to some degree by top-down semantic support (Roberts et al. 2010) which nonwords, by definition, do not possess.

The primary systems view originated in the context of connectionist triangle models of reading aloud (Seidenberg and McClelland 1989; Plaut et al. 1996; Welbourne and Lambon Ralph 2007; Woollams et al. 2007; Welbourne et al. 2011) which focussed primarily on the cognitive-computational aspects of the theory and abstracted away from the specifics of neural implementation. More recently, 'neurocomputational' connectionist models have begun to incorporate neural constraints into the processing architecture whilst maintaining the requirement to explore the key cognitive principles and to generate detailed behavioural data.
Accordingly, such models offer the chance to explore the bridge between neural, systems-level processes and higher cognitive behaviour. Recent prominent examples of this approach include explorations of the relative contributions of the dorsal and ventral language pathways in normal and aphasic language processing (Ueno et al. 2011) and the role of graded lateralisation of visual function in the posterior fusiform in pure alexia (Plaut and Behrmann 2011). The combined neural and behavioural exploration of the primary systems framework undertaken in this study provides important information on how to extend these neurocomputational approaches to model normal and impaired reading.

The multi-dimensional correlation-based approach which we have applied to reading in this study allows us to consider associations between the neural bases of distinct but hypothetically related abilities, in contrast to a more traditional focus on dissociating task-specific brain regions. Our study has allowed us to harness lesion data to reveal the basis for behavioural correlations between spoken language impairments and reading deficits in terms of shared brain regions that support both abilities. This approach has the potential to be applied to other domains of higher cognition, where data reduction techniques can offer behavioural variables suitable for use in lesion-symptom analyses of cross-task association. This approach has the advantage of offering control for factors such as global severity, which is a key issue in neurodegenerative conditions (Lambon Ralph et al. 2003) and also neurodevelopmental disorders. Application of the multi-dimensional correlation-based approach to these populations will allow us to understand how task-specific cognitive processes draw on domain-general networks to optimise the efficiency of the neural systems supporting higher cognition.

Acknowledgements We are extremely grateful to Dr. Rebecca Butler for data collection and coding and image processing for some of the patients included in this paper. This work was supported by an MRC programme Grant (MR/R023883/1) and ERC Grant (GAP: 670428-BRAIN2MIND_NEUROCOMP) to MALR, and a Rosetrees Trust Research Grant to MALR, AW, and AH. Correspondence concerning this article may be address to anna.woollams@manchester. ac.uk.

Open Access This article is distributed under the terms of the Creative Commons Attribution 4.0 International License (http://creativeco mmons.org/licenses/by/4.0/), which permits unrestricted use, distribution, and reproduction in any medium, provided you give appropriate credit to the original author(s) and the source, provide a link to the Creative Commons license, and indicate if changes were made. 


\section{References}

Basilakos A, Fillmore PT, Rorden C, Guo D, Bonilha L, Fridriksson J (2014) Regional white matter damage predicts speech fluency in chronic post-stroke aphasia. Front Hum Neurosci 8:845

Bates E, Wilson SM, Saygin AP, Dick F, Sereno MI, Knight RT, Dronkers NF (2003) Voxel-based lesion-symptom mapping. Nat Neurosci 6:448-450

Binder JR, Westbury CF, McKiernan KA, Possing ET, Medler DA (2005) Distinct brain systems for processing concrete and abstract concepts. J Cogn Neurosci 17:905-917

Binney RJ, Embleton KV, Jefferies E, Parker GJM, Lambon Ralph MA (2010) The ventral and inferolateral aspects of the anterior temporal lobe are crucial in semantic memory: evidence from a novel direct comparison of distortion-corrected fMRI, rTMS, and semantic dementia. Cereb Cortex 20:2728-2738

Boukrina O, Barrett AM, Alexander EJ, Yao B, Graves WW (2015) Neurally dissociable cognitive components of reading deficits in subacute stroke. Front Hum Neurosci 29:Article 298

Bozeat S, Lambon Ralph MA, Patterson K, Garrard P, Hodges JR (2000) Non-verbal semantic impairment in semantic dementia. Neuropsychologia 38:1207-1215

Brookshire CE, Wilson JP, Nadeau SE, Gonzalez Rothi LJ, Kendall DL (2014) Frequency, nature, and predictors of alexia in a convenience sample of individuals with chronic aphasia. Aphasiology 28:1464-1480

Burgess PW, Shallice T (1997) The Hayling and Brixton tests. Thames Valley Test Company, Bury St Edmunds

Butler RA, Ralph MAL, Woollams AM (2014) Capturing multidimensionality in stroke aphasia: mapping principal behavioural components to neural structures. Brain 137:3248-3266

Catani M, ffytche DH (2005) The rises and falls of disconnection syndromes. Brain 128:2224-2239

Catani M, Mesulam M (2008) What is a disconnection syndrome? Cortex 44:911-913

Catani M, Jones DK, ffytche DH (2005) Perisylvian language networks of the human brain. Ann Neurol 57:8-16

Catani M, Mesulam MM, Jakobsen E, Malik F, Martersteck A, Wieneke C, Thompson CK, Thiebaut De Schotten M, Dell'Acqua F, Weintraub S, Rogalski E (2013) A novel frontal pathway underlies verbal fluency in primary progressive aphasia. Brain 136:2619-2628

Cattinelli I, Borghese NA, Gallucci M, Paulesu E (2013) Reading the reading brain: a new meta-analysis of functional imaging data on reading. J Neurolinguist 26:214-238

Crisp J, Lambon Ralph MA (2006) Unlocking the nature of the phonological-deep dyslexia continuum: the keys to reading aloud are in phonology and semantics. J Cogn Neurosci 18:348-362

Cumming TB, Patterson K, Verfaellie MM, Graham KS (2006) One bird with two stones: abnormal word length effects in pure alexia and semantic dementia. Cogn Neuropsychol 23:1130-1161

Dick AS, Bernal B, Tremblay P (2014) The language connectome: new pathways, new concepts. Neuroscientist 20:453-467

Duffau H, Gatignol P, Mandonnet E, Peruzzi P, Tzourio-Mazoyer N, Capelle L (2005) New insights into the anatomo-functional connectivity of the semantic system: a study using cortico-subcortical electrostimulations. Brain 128:797-810

Duffau H, Gatignol P, Moritz-Gasser S, Mandonnet E (2009) Is the left uncinate fasciculus essential for language? A cerebral stimulation study. J Neurol 256:382-389

Fiez JA, Tranel D, Seager-Frerichs D, Damasio H (2006) Specific reading and phonological processing deficits are associated with damage to the left frontal operculum. Cortex 42:624-643
Fujii M, Maesawa S, Motomura K, Futamura M, Hayashi Y, Koba I, Wakabayashi T (2015) Intraoperative subcortical mapping of a language-associated deep frontal tract connecting the superior frontal gyrus to Broca's area in the dominant hemisphere of patients with glioma. J Neurosurg 122:1390-1396

Goodglass H, Kaplan E (1983) The assessment of aphasia and related disorders, 2nd edn. Lea \& Febiger, Philadelphia

Goodglass H, Kaplan E, Barresi B (2000) The assessment of aphasia and related disorders, 3rd edn. Pro-Ed, Austin

Halai A, Woollams AM, Lambon Ralph MA (2017) Using principal component analysis to capture individual differences within a unified neuropsychological model of chronic post-stroke aphasia: revealing the unique neural correlates of speech fluency, phonology and semantics. Cortex 86:275-289

Henry ML, Beeson PM, Alexander GE, Rapcsak SZ (2012) Written language impairments in primary progressive aphasia: a reflection of damage to central semantic and phonological processes. J Cogn Neurosci 24:261-275

Hoffman P, Lambon Ralph MA, Woollams AM (2015a) Triangulation of the neurocomputational architecture underpinning reading aloud. Proc Natl Acad Sci USA 112:E3719-E3728

Hoffman P, Binney RJ, Lambon Ralph MA (2015b) Differing contributions of inferior prefrontal and anterior temporal cortex to concrete and abstract conceptual knowledge. Cortex 63:250-266

Jefferies E, Patterson K, Jones RW, Lambon Ralph MA (2009) Comprehension of concrete and abstract words in semantic dementia. Neuropsychology 23:492-499

Kaplan E, Goodglass H, Weintraub S (1983) Boston naming test. Lea \& Febiger, Philadelphia

Kay J, Lesser R, Coltheart M (1992) PALPA: psycholinguistic assessments of language processing in aphasia. Erlbaum, Hove

Kinoshita M, de Champfleur NM, Deverdun J, Moritz-Gasser S, Herbet G, Duffau H (2015) Role of fronto-striatal tract and frontal aslant tract in movement and speech: an axonal mapping study. Brain Struct Funct 220:3399-3412

Kronfeld-Duenias V, Amir O, Ezrati-Vinacour R, Civier O, BenShachar M (2016) The frontal aslant tract underlies speech fluency in persistent developmental stuttering. Brain Struct Funct 221:365-381

Lambon Ralph MA, Moriarty L, Sage K (2002) Anomia is simply a reflection of semantic and phonological impairments: evidence from a case-series study. Aphasiology 16:56-82

Lambon Ralph MA, Patterson K, Graham NL, Dawson K, Hodges JR (2003) Homogeneity and heterogeneity in mild cognitive impairment and Alzheimer's disease: a cross-sectional and longitudinal study of 55 cases. Brain 126:2350-2362

Mirman D, Graziano KM (2013) The neural basis of inhibitory effects of semantic and phonological neighbors in spoken word production. J Cogn Neurosci 25:1504-1516

Nichols TE, Holmes AP (2002) Nonparametric permutation tests for functional neuroimaging: a primer with examples. Hum Brain Mapp 15:1-25

Nichols T, Brett M, Andersson J, Wager T, Poline JB (2005) Valid conjunction inference with the minimum statistic. NeuroImage 25:653-660

Paivio A (2010) Dual coding theory and the mental lexicon. Ment Lex $5: 205-230$

Parker GJM, Luzzi S, Alexander DC, Wheeler-Kingshott CAM, Ciccarelli O, Lambon Ralph MA (2005) Lateralization of ventral and dorsal auditory-language pathways in the human brain. NeuroImage 24:656-666

Pattamadilok C, Bulnes LC, Devlin JT, Bourguignon M, Morais J, Goldman S, Kolinsky R (2015) How early does the brain distinguish between regular words, irregular words, and pseudowords during the reading process? Evidence from neurochronometric TMS. J Cogn Neurosci 27:1259-1274 
Patterson K, Lambon Ralph MA (1999) Selective disorders of reading? Curr Opin Neurobiol 9:235-239

Phan TG, Donnan GA, Wright PM, Reutens DC (2005) A digital map of middle cerebral artery infarcts associated with middle cerebral artery trunk and branch occlusion. Stroke 36:986-991

Plaut DC, Behrmann M (2011) Complementary neural representations for faces and words: a computational exploration. Cogn Neuropsychol 28:251-275

Plaut DC, Shallice T (1993) Deep dyslexia: a case study of connectionist neuropsychology. Cogn Neuropsychol 10:377-500

Plaut DC, McClelland JL, Seidenberg MS, Patterson K (1996) Understanding normal and impaired word reading: computational principles in quasi-regular domains. Psychol Rev 103:56-115

Price CJ (2012) A review and synthesis of the first 20 years of PET and fMRI studies of heard speech, spoken language and reading. NeuroImage 62:816-847

Rapcsak SZ, Beeson PM, Henry ML, Leyden A, Kim E, Rising K, Andersen S, Cho H (2009) Phonological dyslexia and dysgraphia: cognitive mechanisms and neural substrates. Cortex 45:575-591

Raven JC (1962) Coloured progressive matrices, sets A, Ab, B. H.K, Lewis, London

Richardson JD, Fillmore P, Rorden C, Lapointe LL, Fridriksson J (2012) Re-establishing Broca's initial findings. Brain Lang 123:125-130

Ripamonti E, Aggujaro S, Molteni F, Zonca G, Frustaci M, Luzzatti C (2014) The anatomical foundations of acquired reading disorders: a neuropsychological verification of the dual-route model of reading. Brain Lang 134:44-67

Roberts DJ, Lambon Ralph MA, Woollams AM (2010) When does less yield more? The impact of severity upon implicit recognition in pure alexia. Neuropsychologia 48:2437-2446

Roberts DJ, Woollams AM, Kim E, Beeson PM, Rapcsak SZ, Lambon Ralph MA (2013) Efficient visual object and word recognition relies on high spatial frequency coding in the left posterior fusiform gyrus: evidence from a case-series of patients with ventral occipito-temporal cortex damage. Cereb Cortex 23:2568-2580

Sabsevitz DS, Medler DA, Seidenberg M, Binder JR (2005) Modulation of the semantic system by word imageability. NeuroImage 27:188-200

Saur D, Kreher BW, Schnell S, Kümmerer D, Kellmeyer P, Vry M-S, Umarova R, Musso M, Glauche V, Abel S, Huber W, Rijntjes M, Hennig J, Weiller C (2008) Ventral and dorsal pathways for language. Proc Natl Acad Sci USA 105:18035-18040

Schmahmann JD, Pandya DN (2008) Disconnection syndromes of basal ganglia, thalamus, and cerebrocerebellar systems. Cortex 44:1037-1066

Schwartz MF, Faseyitan OK, Kim J, Coslett HB (2012) The dorsal stream contribution to phonological retrieval in object naming. Brain 135:3799-3814

Sebastian R, Gomez Y, Leigh R, Davis C, Newhart M, Hillis AE (2014) The roles of occipitotemporal cortex in reading, spelling, and naming. Cogn Neuropsychol 31:511-528

Seghier ML, Ramlackhansingh A, Crinion JT, Leff AP, Price CJ (2008) Lesion identification using unified segmentation-normalisation models and fuzzy clustering. NeuroImage 41:1253-1266

Seidenberg MS, McClelland JL (1989) A distributed, developmental model of word recognition and naming. Psychol Rev 96:523-568

Sliwinska MW, James A, Devlin JT (2015) Inferior parietal lobule contributions to visual word recognition. J Cogn Neurosci 27:593-604

Swinburn K, Porter G, Howard D (2005) Comprehensive aphasia test. Psychology Press, Hove

Taylor JSH, Rastle K, Davis MH (2013) Can cognitive models explain brain activation during word and pseudoword reading?
A meta-analysis of 36 neuroimaging studies. Psychol Bull 139:766-791

Tree JJ (2008) Two types of phonological dyslexia—a contemporary review. Cortex 44:698-706

Tree JJ, Kay J (2006) Phonological dyslexia and phonological impairment: an exception to the rule? Neuropsychologia 44:2861-2873

Tyler LK, Marslen-Wilson WD, Stamatakis EA (2005) Dissociating neuro-cognitive component processes: voxel-based correlational methodology. Neuropsychologia 43:771-778

Ueno T, Saito S, Rogers TT, Lambon Ralph MA (2011) Lichtheim 2: synthesizing aphasia and the neural basis of language in a neurocomputational model of the dual dorsal-ventral language pathways. Neuron 72:385-396

Vassal F, Boutet C, Lemaire JJ, Nuti C (2014) New insights into the functional significance of the frontal aslant tract: an anatomofunctional study using intraoperative electrical stimulations combined with diffusion tensor imaging-based fiber tracking. Br J Neurosurg 28:685-687

Vigneau M, Beaucousin V, Hervé PY, Duffau H, Crivello F, Houdé O, Mazoyer B, Tzourio-Mazoyer N (2006) Meta-analyzing left hemisphere language areas: phonology, semantics, and sentence processing. NeuroImage 30:1414-1432

Visser M, Jefferies E, Lambon Ralph MA (2010a) Semantic processing in the anterior temporal lobes: a meta-analysis of the functional neuroimaging literature. J Cogn Neurosci 22:1083-1094

Visser M, Embleton KV, Jefferies E, Parker GJM, Lambon Ralph MA (2010b) The inferior, anterior temporal lobes and semantic memory clarified: novel evidence from distortion-corrected fMRI. Neuropsychologia 48:1689-1696

Wechsler D (1987) Manual for the Wechsler Memory Scale-revised. Psychological Corporation, San Antonio

Welbourne SR, Lambon Ralph MA (2007) Using parallel distributed processing models to simulate phonological dyslexia: the key role of plasticity-related recovery. J Cogn Neurosci 19:1125-1139

Welbourne SR, Woollams AM, Crisp J, Ralph MAL (2011) The role of plasticity-related functional reorganization in the explanation of central dyslexias. Cogn Neuropsychol 28:65-108

Westbury C, Moroschan G (2009) Imageability $\times$ phonology interactions during lexical access: effects of modality, phonological neighbourhood, and phonological processing efficiency. Ment Lex $4: 115-145$

Wilke M, de Haan B, Juenger H, Karnath HO (2011) Manual, semiautomated, and automated delineation of chronic brain lesions: a comparison of methods. NeuroImage 56:2038-2046

Wilson SM, Brambati SM, Henry RG, Handwerker DA, Agosta F, Miller BL, Wilkins DP, Ogar JM, Gorno-Tempini ML (2009) The neural basis of surface dyslexia in semantic dementia. Brain 132:71-86

Wilson MA, Joubert S, Ferré P, Belleville S, Ansaldo AI, Joanette Y, Rouleau I, Brambati SM (2012) The role of the left anterior temporal lobe in exception word reading: reconciling patient and neuroimaging findings. NeuroImage 60:2000-2007

Wise RJS (2003) Language systems in normal and aphasic human subjects: functional imaging studies and inferences from animal studies. Br Med Bull 65:95-119

Woollams AM (2014) Connectionist neuropsychology: uncovering ultimate causes of acquired dyslexia. Philos Trans R Soc B Biol Sci 369:20120398

Woollams AM, Lambon Ralph MA, Plaut DC, Patterson K (2007) SD-squared: on the association between semantic dementia and surface dyslexia. Psychol Rev 114:316-339

Woollams AM, Lambon Ralph MA, Plaut DC, Patterson K (2010) SDsquared revisited: reply to Coltheart, Tree, and Saunders (2010). Psychol Rev 117:273-281 